PROCEEDINGS OF THE

AMERICAN MATHEMATICAL SOCIETY

Volume 48, Number 1, March 1975

\title{
CONTINUOUS METRIC PROJECTIONS
}

\author{
JOSEPH M. LAMBERT
}

ABSTRACT. An example is given of a reflexive, rotund Banach space whose dual space is not Fréchet differentiable such that every metric projection onto closed subspaces is norm continuous. This example shows that several published conjectures on necessary and sufficient conditions for a reflexive, rotund Banach space to have norm continuous metric projections onto all closed subspaces are incorrect.

Introduction. If $X$ is a reflexive, rotund Banach space, every closed subspace of $X$ is a unique best approximation subspace, called a Chebyshev subspace. If $M$ is a closed subspace of $X$, the metric projection $P_{M}$ associated with $M$ is defined via inf ${ }_{m} \in M\|x-m\|=\left\|x-P_{m}(x)\right\|$. An open question in best approximation theory is to find necessary and sufficient conditions on a reflexive, rotund Banach space to insure that every closed subspace has a norm continuous metric projection associated with it. In [5], the conjecture was that every reflexive and rotund Banach space had this property. In [4], this was later amended to be that Banach spaces with a Fréchet smooth dual space had this property. In [4], it was also conjectured that if the canonical duality map from $X^{*}$ to $X$, restricted to $M^{\perp}=\left\{x^{*} \in X^{*} \mid x^{*}(m)=0, \forall m \in M\right\}$, was norm continuous, then $P_{M}$ was norm continuous. It was verified for subspaces $M$ of finite codimension in [4]. In [1], the study of bounded compactness led to the continuity of $P_{M}$ for those $M$ such that $\operatorname{ker} P_{M}=\left\{x \in X \mid P_{M}(x)\right.$ $=\theta\}$ was boundedly compact. Further, if the codimension of $M$ was finite, then the bounded compactness of $\operatorname{ker} P_{M}$ was a necessary and sufficient condition that $P_{M}$ be norm continuous.

In this paper, we show that there exists a reflexive, rotund Banach space whose dual is not Fréchet smooth and yet every metric projection is norm continuous. This is accomplished by closely examining the space $X$ exhibited by Klee in [6], where $X$ is a renorm of $l_{2}$, which is reflexive, Gâteaux smooth at all points of the unit ball except $\left\{-\delta_{0}, \delta_{0}\right\}$. By well-known duality relationships, $X^{*}$ is a reflexive, rotund Banach space. It is this space which will be

Received by the editors September 29, 1972 .

AMS (MOS) subject classifications (1970). Primary 41A65; Secondary 46B10.

Key words and phrases. Abstract approximation, metric projections, best approximation maps. 
shown to have the desired properties. In $\$ 1$ we will recall the Klee space, following the notation in [6]. In $\$ 2$ we recall the terminology of abstract approximation and show that the dual of the Klee space provides an example which exhibits a reflexive, rotund Banach space whose dual is not Fréchet smooth and yet every metric projection onto closed subspaces is norm continuous. In $\$ 3$, we show that the theorems in [4] and [1] cannot be extended when the dimension requirements on $M$ are removed.

In this paper $X$ will denote a Banach space and $X^{*}$ its continuous dual. $U(X)$ and $S(X)$ will denote the closed unit ball in $X$ and its boundary, respectively. All other notation will correspond to that in [2]. A source for the theory of metric projections is [3, \$32].

1. The Klee space. A renorm of $l_{2}$ is given in [6] as follows. An element $x$ is in $l_{2}$ if and only if $x=\left\{x_{i}\right\}_{i=0}^{\infty}$ such that $\sum_{i=0}^{\infty}\left|x_{i}\right|^{2}<\infty$. Let $V=\left\{x \in l_{2} \mid x_{0}=0\right\}$. Let $U_{V}$ and $S_{V}$ denote the unit ball and its boundary, respectively, of the subspace $V$. For each bounded sequence $a=\left\{a_{i}\right\}_{i=1}^{\infty}$, let $T_{a}$ denote the linear transformation of $V$ into $V$ defined by $T_{a}(x)=$ $\left(0, a_{1} x_{1}, a_{2} x_{2}, \cdots\right), x \in V$. For each $\lambda \in[-1,1]$ and each sequence $\eta=$ $\left(\eta_{1}, \eta_{2}, \ldots\right)$ of even functions on $[-1,1]$ to $[0,1]$ with $\eta_{i}(0)=1$ for all $i$, let $\eta(\lambda)=\left(\eta_{1}(\lambda), \eta_{2}(\lambda), \ldots\right)$. Then let

$$
U_{\eta}=\bigcup_{|\lambda| \leq 1}\left[\lambda \delta_{0}+T_{\eta(\lambda)} U_{V}\right] \text { and } S_{\eta}=\bigcup_{|\lambda| \leq 1}\left[\lambda \delta_{0}+T_{\eta(\lambda)} S_{V}\right]
$$

where $\delta_{0}=(1,0,0 \cdots)$. Further let $U$ be the convex closure of $U_{\eta}$ and let $S$ be the boundary of $U$. Klee prescribed the following conditions on $\left\{\eta_{i}\right\}$ to obtain the required smoothness conditions.

K1. $\eta_{i}$ is continuous and concave, with $\eta_{i}(0)=1, \eta_{i}\left(1-\epsilon_{i}\right)=2 \epsilon_{i}$ and $\eta_{i}(1)=0$ for all $i$.

K2. $\eta_{i}$ is differentiable on $[0,1]$ with $\eta_{i}^{\prime}(0)=0$ and $\eta_{i}^{\prime}\left(1-\epsilon_{i}\right)=-1$ for all $i$.

K3. $\eta_{i}$ has a vertical tangent at 1 , i.e. $\lim _{\lambda \rightarrow 1} \eta_{i}^{\prime}(\lambda)=-\infty$ for all $i$.

K4. $\left\{\epsilon_{i}\right\}_{i=1}^{\infty}$ is a sequence of real numbers such that $\epsilon_{i} \in[0,1 / 6)$ with $\epsilon_{i} \rightarrow 0$ as $i \rightarrow \infty$.

We wish to place a further restriction on the $\eta_{i}$ to facilitate computational problems.

K5. For all $i, \eta_{i}(\lambda) \geq\left(1-\lambda^{2}\right)^{1 / 2}$.

The gauge of $U, \rho_{U}$ is the renorm of $l_{2}$. The remaining facts to be recalled for our investigation are:

Ka. $U_{\eta}=\left\{-\delta_{0}, \delta\right\} \cup\left\{x \in l_{2}|| x_{0} \mid<1\right.$ and $\left.\sum_{i=1}^{\infty}\left(x_{i} / \eta_{i}\left(x_{0}\right)\right)^{2} \leq 1\right\}$.

Kb. The intersection of $S_{\eta}$ with the half plane $G_{s}=L+[0, \infty) s, s \in S_{V}$, $L=\left\{\lambda \delta_{0} \mid-\infty<\lambda<\infty\right\}$, is given by $\left\{\lambda \delta_{0}+\tau_{s}(\lambda) \cdot s|| \lambda \mid \leq 1\right\}$, where $\tau_{s}(\lambda)$ 
is the positive solution of $\sum_{i=1}^{\infty}\left[\tau_{s}(\lambda) s_{i} / \eta_{i}(\lambda)\right]^{2}=1$.

Kc. The Klee space $\left(X, \rho_{U}\right)$ is reflexive, Gâteaux smooth at all points of $U$ and Fréchet smooth at all points of $U$ except $\left\{-\delta_{0}, \delta_{0}\right\}$.

2. The example. Recall that if $M$ is a closed subspace of a Banach space $Z, d(z, M)=\inf \{\|z-m\| \mid m \in M\}$. Let $P_{M}(z)=\{m \in M \mid\|z-m\|=$ $d(z, M)\}$. The set valued mapping $z \rightarrow P_{M}(z)$ is called the metric projection of $Z$ onto $M$. If $P_{M}$ is a single valued mapping, $M$ is called a Chebyshev subspace. The set $\left\{z \in Z \mid P_{M}(z)=\theta\right\}$ will be denoted $\operatorname{ker} P_{M}$.

Let $(Y,\|\cdot\|)$ denote $\left(X, \rho_{U}\right)^{*}$ where $\left(X, \rho_{U}\right)$ is the Klee space described in $\S 1$. Since $\left(X, \rho_{U}\right)$ was reflexive and smooth, one has $(Y,\|\cdot\|)$ is reflexive and rotund. Let $M$ be any Chebyshev subspace of $Y$. Let $\psi_{M}$ : $Y \backslash M \rightarrow S\left(\operatorname{ker} P_{M}\right)$ via $\psi_{M}(y)=\left(y-P_{M}(y)\right) /\left\|y-P_{M}(y)\right\|$. Further, let $T:$ $Y^{*} \rightarrow Y$ be the canonical duality given by $T y^{*}=\left\{y \in Y \mid y\left(y^{*}\right)=\|y\|\left\|y^{*}\right\|\right\}$. Theorem 13 in [14] states that if $T$ is continuous at $f \in S\left(Y^{*}\right) \cap M^{\perp}$, then $P_{M}$ is continuous at all points of the set $\psi_{M}^{-1}(T(f))$. Since $Y^{*}=X$, the Klee space $T$ is continuous at all points of $S\left(Y^{*}\right)$ except $\left\{-\delta_{0}, \delta_{0}\right\}$. Since the continuity of $P_{M}$ at all points is equivalent to the continuity of $P_{M}$ at all points in $\operatorname{ker} P_{M}$, we need only study those points $x_{0}$ and those subspaces $M$, such that $\left(x_{0}-P_{M} x_{0}\right) /\left\|x_{0}-P_{M} x_{0}\right\| \in T\left( \pm \delta_{0}\right)$, since $P_{M}$ would be continuous at all other points. In particular, $\delta_{0} \in M^{\perp}$ for such $x_{0}$ and $M$. Thus if $\delta_{0} \notin M^{\perp}, P_{M}$ is norm continuous. We wish to show every $P_{M}$ is norm continuous. Thus we consider those subspaces $M$ such that $\delta_{0} \in M^{\perp}$. We use the fact that since $\left(X, \rho_{U}\right)$ is a renorm of $l_{2}$, $(Y,\|\|)$ can be considered a renorm of $l_{2}$.

Lemma 2.1. Given $M$, a Chebychev subspace of $Y$ such that $\delta_{0} \epsilon$ $M^{\perp} \subset X$, then $M^{\perp}$ taken as a set in $Y$ is contained in $\operatorname{ker} P_{M}$.

Proof. Let $z$ be in $S\left(M^{\perp}\right) \subset Y$. One must show that $z$ is in $\operatorname{ker} P_{M}$. Now $z$ acts as a linear functional on $X=Y^{*}$ and since $X$ is reflexive, $z$ attains its norm on $U$. In particular, by use of the Krein-Milman theorem, $z$ must attain its norm at an extreme point and hence at a point of $S_{\eta}$. Thus there exists $u \in S_{\eta}$ such that $(u, z)=1$. If $u \in S_{\eta} \cap M^{\perp} \subset X$ then $z \in T u \subset \operatorname{ker} P_{M}$, since $T\left(M^{\perp}\right)=\operatorname{ker} P_{M}$, and the result follows. If for all $u \in S_{\eta} \cap M^{\perp},(u, z) \neq 1$, choose any $u \in S_{\eta}$ such that $(u, z)=1$. By previous remarks in $\S 1, u$ has the form $u=\lambda \delta_{0}+\tau_{y}(\lambda) y, y \in s_{V}, \lambda \neq 1$. But $y$ can be written as $y=x+w$, with $x$ in $M^{\perp}$ and $w$ in $M$ considered as sets in $X$ since $X$ is a renorm of $l_{2}$. Since $\delta_{0} \in M^{\perp} \subset X, M \subset V \subset Y$. Thus $w$ is in $V$ and hence $x$ is in $V$. Now, 


$$
(u, z)=\left(\lambda \delta_{0}+\tau_{y}(\lambda)(x+w), z\right)=\left(\lambda \delta_{0}+\tau_{y}(\lambda) x, z\right)
$$

since $z$ is in $M^{\perp}$. We claim $\rho_{U}\left(\lambda \delta_{0}+\tau_{y}(\lambda) x\right)<1$. This follows since

$$
\begin{aligned}
1 & =\rho_{U}(u)=\sum_{i=1}\left[\frac{\tau_{y}(\lambda) y_{i}}{\eta_{i}(\lambda)}\right]^{2}=\sum_{i=1} \frac{\tau_{y}^{2}(y)\left(x_{i}+w_{i}\right)^{2}}{\eta_{i}(\lambda)^{2}} \\
& =\sum_{i=1} \tau_{y}^{2}(y) \frac{x_{i}^{2}+2 x_{i} w_{i}+w_{i}^{2}}{\eta_{i}(\lambda)^{2}}=\sum_{i=1} \tau_{y}^{2}(\lambda) \frac{x_{i}^{2}+w_{i}^{2}}{\eta_{i}(\lambda)^{2}} .
\end{aligned}
$$

The last inequality follows from

$$
\left|\sum_{i=1} \frac{x_{i} w_{i}}{\eta_{i}(\lambda)^{2}}\right| \leq\left|\frac{\sum x_{i} w_{i}}{1-\lambda^{2}}\right|=0
$$

since $(x, w)=\Sigma x_{i} w_{i}=0$ because $x \in M^{\perp} \cap V, w \in M \cap V$, and K5. Thus

$$
\sum_{i=1} \frac{\tau_{y}^{2}(\lambda) x_{i}^{2}}{\eta_{i}(-1)^{2}}=1-\sum_{i=1} \frac{\tau_{y}^{2}(\lambda) w_{i}^{2}}{\eta_{i}(\lambda)^{2}}=k .
$$

Since $u$ is not an element of $M^{\perp}, w \neq \theta$, and hence $k<1$. Thus $\rho_{U}\left(\lambda \delta_{0}+\tau_{y}(\lambda)_{x}\right)=k<1$. We remark that if $k=0$ then $x=\theta$ and $\lambda=1$, implying $z=\delta_{0}$ and $u=\delta_{0}$, a contradiction. By considering $\alpha=$ $k^{-1}\left(\lambda \delta_{0}+\tau_{y}(\lambda) x\right)$ one has $\rho_{U}(\alpha)=1$ and $\alpha \in M^{\perp}$. But $(\alpha, z)=$ $k^{-1}\left(\lambda \delta_{0}+\tau_{y}(\lambda) x, z\right)=k^{-1}>1$. This contradicts $\|z\|=1$. Thus there exists $u \in S_{\eta} \cap M^{\perp}$ such that $z \in T u$. Therefore, $M^{\perp} \subset \operatorname{ker} P_{M}$. Q.E.D.

By [5, Theorem 3], if ker $P_{M}$ contains a subspace $N$ such that $M+N$ $=Z$, the entire Banach space, then $P_{M}: Z \rightarrow M$ is linear and hence continuous. Since $Y$ is a renorm of $l_{2}$, one can write $Y=M+M^{\perp}$. The above remarks coupled with Lemma 2.1 yield that $P_{M}$ is continuous whenever $\delta_{0} \in M^{\perp}$. The initial remarks in $\$ 2$ showed that $P_{M}$ is continuous whenever $\delta_{0} \notin M^{\perp}$. Hence, we have the following

Theorem 2.1. There exists a reflexive, rotund Banach space $Y$ such that $Y^{*}$ is not Fréchet smooth, but $P_{M}$ is norm continuous for all closed subspaces $M$.

This example shows that the conjecture in [4], that the necessary and sufficient condition for a reflexive, rotund Banach space to have the metric projections onto all closed subspaces continuous, be that the dual space be Fréchet smooth, is incorrect. 
3. Further applications as counterexamples. In [4], Theorem 14 states: If $X$ is a reflexive, rotund Banach space, $M$ a Chebyshev subspace of finite codimension and if $T$ is the canonical duality map $T: X^{*} \rightarrow X^{* *}=X$, then $T / M^{\perp}$ is norm continuous if and only if $P_{M}$ is norm continuous.

It was conjectured that this theorem would also be valid without any dimension requirements on $M$. The following example shows that this cannot be the case.

Example 3.1. Let $X$ be the Klee space, $Y$ its dual as above. Let $M \subset$ $Y$ be defined as $M=$ closed $\operatorname{span}\left\{\delta_{2 j} \mid j=1,2, \ldots\right\}$ where $\delta_{j}$ are the usual basis vectors in $l_{2}$. Clearly the closed span of $\left\{\delta_{0}, \delta_{2 j+1} \mid j=0,1,2 \cdots\right\}$ is contained in $M^{\perp}$. Consider the sequence $\left\{x_{2 i+1}\right\}$ in $S\left(M^{\perp}\right)$ defined via $x_{2 i+1}=\left(1-\epsilon_{2 i+1}\right) \delta_{0}+2 \epsilon_{2 i+1} \delta_{2 i+1}$ where the $\left\{\epsilon_{i}\right\}$ are as in $K 4$ in $\$ 1$. It is easily seen that $x_{2 i+1} \rightarrow \delta_{0}$. By an elementary calculation $T\left(x_{2 i+1}\right)=$ $\left(\delta_{0}+\delta_{2 i+1}\right) /\left(1+\epsilon_{2 i+1}\right)$. Hence $T\left(x_{2 i+1}\right)$ converges weakly to $\delta_{0}$, but not in norm. Hence $T / M^{\perp}$ is not continuous, but by the work of the previous section $P_{M}$ is linear and continuous.

A set is boundedly compact if every bounded sequence has a convergent subsequence. In [1], Theorem 8 states: If $X$ is a normed linear space, $M$ a Chebyshev subspace of finite codimension, then $\operatorname{ker} P_{M}$ is boundedly compact if and only if $P_{M}$ is norm continuous.

It was conjectured that the theorem continued to be valid without restriction on the dimension of M. Using Example 3.1, we can show that the theorem cannot be extended. The set $\left\{T\left(x_{2 i}\right)\right\}_{i=0}^{\infty}$ consists of elements of norm one in ker $P_{M}$. However, there does not exist a norm convergent subsequence, and yet, $P_{M}$ is norm continuous.

It should be noted that B. Kripke of Ohio State University, in an unpublished result, has found an example of a Hilbert space which can be renormed with a rotund norm so as to contain a closed subspace $M$ such that $P_{M}$ is not norm continuous.

Finally in [7], Ošman has announced necessary and sufficient conditions for all metric projections to be continuous. These conditions resemble conditions implying Fréchet smoothness of the dual space but are slightly weaker. The example in $\$ 2$ gives a concrete example of such a space.

\section{REFERENCES}

1. E. Cheney and D. Wulbert, The existence and unicity of best approximations, Math. Scand. 24 (1969), 113-140. MR 41 \#5857.

2. N. Dunford and J. T. Schwartz, Linear operators. I: General theory, Pure and Appl. Math., vol. 7, Interscience, New York, 1958. MR 22 \#8302.

3. R. Holmes, A course on optimization and best approximation, Lecture Notes in Math., vol. 257, Springer-Verlag, New York, 1972. 
4. R. Holmes, On the continuity of best approximation operators, Sympos. on Infinite Dimensional Topology, Ann. of Math. Studies, vol. 69, Princeton Univ. Press, Princeton, N. J., 1972.

5. R. Holmes and B. Kripke, Smoothness of approximation, Michigan Math. J. 15 (1968), 225-248. MR 37 \#483.

6. V. Klee, Two renorming constructions related to a question of Anselone, Studia Math. 33 (1969), 231-242. MR 40 \#1756.

7. E. Ošman, On continuity of metric projection onto some classes of subspaces in a Banach space, Dokl. Akad. Nauk SSSR 195 (1970), 555-557 = Soviet Math. Dokl. 11 (1970), 1521-1523. MR $42 \# 8251$.

DEPARTMENT OF MATHEMATICS, PENNSYLVANIA STATE UNIVERSITY, YORK, PENNSYLVANIA 17403 